\title{
Method to improve degraded range resolution due to non-ideal factors in FMCW radar
}

\author{
You-Sun Won ${ }^{1}$, Dongseung Shin ${ }^{1}$, Sohee Jung ${ }^{1}$, Jae-Ho Lee ${ }^{1}$, \\ Cheolhyo Lee ${ }^{1}$, Miryong Park ${ }^{1}$, Yunjeong Song ${ }^{1}$, Kiyoung Moon ${ }^{1}$, \\ and Dong-Wook Seo ${ }^{2 \mathrm{a}}$ \\ ${ }^{1}$ Smart Vehicle Research Section, Electronics and Telecommunications Research \\ Institute, Daegu, 42994, Republic of Korea \\ 2 Dept. of Radio Communication Engineering, Korea Maritime and Ocean \\ University, Busan, 49112, Republic of Korea \\ a)dwseo@kmou.ac.kr, Corresponding Author
}

\begin{abstract}
This paper reports a method to improve a degraded range resolution for FMCW radar. The proposed post-processing method can achieve an improved range resolution without increasing a signal bandwidth by eliminating factors that can degrade the range resolution based on the non-negative least-squares method. For an FMCW radar adopting the postprocessing method with the center frequency of $76.5 \mathrm{GHz}$ and the signal bandwidth of $200 \mathrm{MHz}$, simulation results show that the degraded range resolution of $160 \mathrm{~cm}$ is improved to $70 \mathrm{~cm}$, and measurements show that two corner reflectors with the radar cross section of $10 \mathrm{dBsm}$ located at $70 \mathrm{~cm}$ range intervals can be distinguished.
\end{abstract}

Keywords: FMCW, radar, range resolution, signal bandwidth, non-negative least-squares method

Classification: Microwave and millimeter-wave devices, circuits, and modules

\section{References}

[1] B. R. Mahafza: Radar Systems Analysis and Design Using MATLAB (CRC Press, FL, Boca Raton, 2000).

[2] C. Lee, et al.: "High resolution LFMCW radar system using model-based beat frequency estimation in cable fault localization," IEICE Electron. Express 11 (2014) 20130768 (DOI: 10.1587/elex.10.20130768).

[3] J. Hong and C. Cheon: "Simple VCO nonlinearity correction algorithm based on EMD in FMCW radar system," Electron. Lett. 52 (2016) 1484 (DOI: 10. 1049/el.2016.1986).

[4] S. O. Piper: "Homodyne FMCW radar range resolution effects with sinusoidal nonlinearities in the frequency sweep," IEEE Int. Radar Conf. Rec. (1995) 563 (DOI: 10.1109/RADAR.1995.522609).

[5] S. Ayhan, et al.: "Impact of frequency ramp nonlinearity, phase noise, and SNR on FMCW radar accuracy," IEEE Trans. Microw. Theory Techn. 64 (2016) 
3290 (DOI: 10.1109/TMTT.2016.2599165).

[6] Z. Xia, et al:: "A novel handheld pseudo random coded UWB radar for human sensing applications," IEICE Electron. Express 11 (2014) 20140981 (DOI: 10. 1587/elex.11.20140981).

[7] Y. Li and S. O'Young: "Method of doubling range resolution without increasing bandwidth in FMCW radar," Electron. Lett. 51 (2015) 933 (DOI: 10. 1049/el.2015.0309).

[8] Y. Won, et al.: "Range resolution improvement of a $24 \mathrm{GHz}$ ISM band pulse radar - a feasibility study,” IEEE Sensors J. 15 (2015) 7142 (DOI: 10.1109/ JSEN.2015.2469154).

[9] F. Ding, et al.: "Aliasing radar receiver in FMICW system," IEICE Electron. Express 7 (2010) 697 (DOI: 10.1587/elex.7.697).

[10] C. L. Lawson and R. J. Hanson: Solving Least-Squares Problems (PrenticeHall, Englewood Cliffs, NJ, USA, 1974).

\section{Introduction}

A frequency-modulated continuous wave (FMCW) radar is a device that outputs object information such as range, velocity, and angle through radar signal processing [1]. Range resolution to detect adjacent objects separately is one of the important performance parameters of FMCW radar [2]. The theoretical range resolution can be calculated with the light velocity and signal bandwidth. However, the actual range resolution is degraded due to non-ideal factors in FMCW radar. Non-ideal factors include the reduced effective modulation bandwidth due to timeof-flight delays, the increased main-lobe null-to-null bandwidth when FFT windows are applied, and non-linearity of frequency sweeps [3, 4, 5]. Various studies have been reported to improve range resolution, such as increasing bandwidth [6], using triangular sweep signal [7], over-sampling [8], and removing aliasing effects [9]. However, since these methods cannot suppress non-ideal effects on range resolution, range resolution can be further improved by compensating for non-ideal factors. In this paper, post-processing based on the non-negative least-squares method (LSM) is applied to compensate non-ideal factors and improve range resolution without increasing signal bandwidth.

\section{Range resolution improvement for FMCW radar}

Fig. 1 shows the time-frequency plot of a sawtooth sweep signal which is mainly used in FMCW radar. When continuous chirp signals with a chirp length $t_{m}$ and a modulation bandwidth $\Delta f$ are transmitted from $\mathrm{FMCW}$ radar, an echo signal reflected from objects is received after a time-of-flight delay $t_{\text {delay }}$. By mixing a received signal with a transmitted signal, a beat signal, which has a frequency difference $f_{\text {beat }}$ between two signals, can be obtained and a range can be calculated.

However, there are several non-ideal factors that degrade the range resolution, such as reduced effective modulation time $t_{\text {meff }}$ or bandwidth $\Delta f_{\text {eff }}$ due to $t_{\text {delay }}$, nonlinearity in the frequency sweep, and FFT window function effects. First, as shown in Fig. 1, the effective modulation time to find $f_{\text {beat }}$ is $t_{\text {meff }}$, which is reduced by $t_{\text {delay }}$ from $t_{m}$, while the effective modulation bandwidth is reduced from 


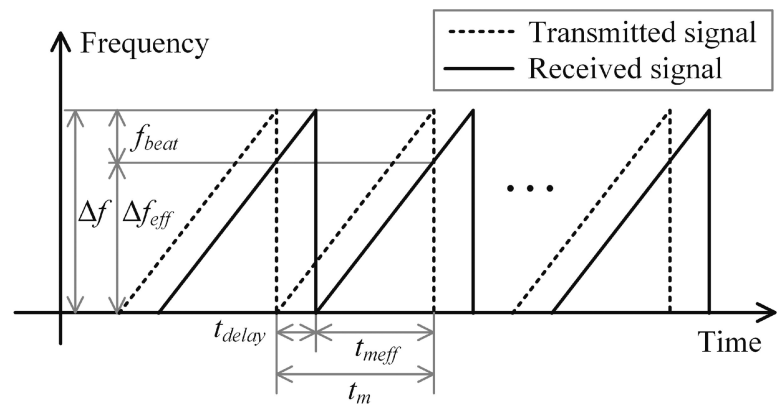

Fig. 1. Time frequency plot of sawtooth sweep signal for FMCW radar

$\Delta f$ to $\Delta f_{\text {eff }}$. Therefore, the range resolution $\Delta R$ given by Eq. (1) is degraded by the ratio of $t_{m e f f}$ to $t_{m}$ [4]:

$$
\Delta R=\frac{c}{2 \Delta f_{\text {eff }}}=\frac{c}{2 \Delta f\left(\frac{t_{\text {meff }}}{t_{m}}\right)},
$$

where $c$ is the light velocity. Next, nonlinearity in frequency sweep means that chirp distortion or discontinuity occurs due to noise or the performance limitations of waveform generators. If the slope of a chirp signal is not linear, it is difficult to distinguish between multiple objects because beat frequency components corresponding to object positions cannot be accurately predicted. Finally, adopting FFT windows in the time domain for range FFT lowers the side-lobe level in the frequency domain, but it broadens the null-to-null bandwidth of the main-lobe spectra [1]. In the frequency domain, the broadened null-to-null bandwidth degrades $\Delta R$ because multiple beat frequency components are overlapped and difficult to distinguish. To improve $\Delta R$ without increasing $\Delta f_{\text {eff }}$, the influence of these non-ideal factors should be reduced.

Fortunately, the reduced $t_{\text {meff }}$, nonlinearity in the frequency sweep, and FFT window function effects can be quantified through design processes and module measurements. Therefore, a high-resolution range profile can be obtained by removing predictable and quantifiable non-ideal factors from measured beat signals. Fig. 2 shows a block diagram of the proposed radar signal processing with post-processing after the conventional range FFT to compensate for degraded $\Delta R$ in FMCW radar. The post-processing can be implemented by solving an equation given in Eq. (2) using the LSM [10]:

$$
\arg \min _{\mathbf{x}}\|\mathbf{D} \mathbf{x}-\mathbf{y}\|_{2}^{2}=\left\|\left[\begin{array}{cccc}
s_{1} & 0 & \cdots & 0 \\
\vdots & s_{1} & & \vdots \\
s_{L} & \vdots & \ddots & 0 \\
0 & s_{L} & & s_{1} \\
\vdots & & \ddots & \vdots \\
0 & \cdots & 0 & s_{L}
\end{array}\right]\left[\begin{array}{c}
x_{1} \\
x_{2} \\
x_{3} \\
\vdots \\
x_{M-1} \\
x_{M}
\end{array}\right]-\left[\begin{array}{c}
y_{1} \\
y_{2} \\
y_{3} \\
\vdots \\
y_{M-1} \\
y_{M}
\end{array}\right]\right\| \text {, }
$$

where $\mathbf{D}$ is the correlation matrix, $\mathbf{x}$ is the estimated discrete range profile, and $\mathbf{y}$ is the range FFT result of measured echo signals at the baseband. $\mathbf{s}=\left[\begin{array}{llll}s_{1} & s_{2} & \cdots & s_{L}\end{array}\right]^{T}$ 


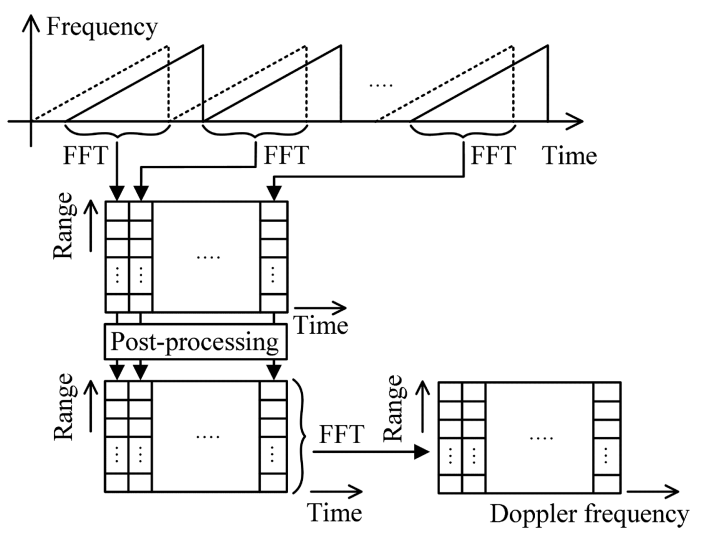

Fig. 2. Block diagram of radar signal processing

is the normalized range FFT result within null-to-null bandwidth $L$ of an estimated beat signal for one object detection that reflects predictable non-ideal effects. The correlation matrix $\mathbf{D}$ consists of $\mathbf{s}$ shifted by one range bin for each column. Here, $M$ is the number of range bins. By solving Eq. (2) based on the LSM, the measured range FFT result is decomposed into peak range bins in $\mathbf{x}$ corresponding to object positions and waveform spectra in $\mathbf{D}$ reflecting degradation information in the frequency domain. Because power spectra are non-negative variables, the nonnegative LSM can be used to obtain $\mathbf{x}$ in Eq. (2) to mitigate overfitting due to random noise.

\section{Simulation and measurement results}

To verify the improvement in the range resolution with the proposed post-processing method, radar signal processing was performed using MATLAB. For radar signal processing based on Eq. (2), $\mathbf{y}$ and $\mathbf{D}$ were generated considering radar parameters and non-ideal factors, and $\mathbf{x}$ was estimated using the non-negative LSM from $\mathbf{y}$ and $\mathbf{D}$. To analyze only the effect on $\Delta R$, it is assumed that objects are stationary. A time-domain beat signal $y_{1}$ for one object with non-linearity noise [4] is given by

$$
y_{1}(t)=c_{0} \cos 2 \pi\left\{\begin{array}{c}
\left(f_{0}+\frac{\Delta f}{2}\right)\left(\frac{2 R}{c}\right)+\frac{\Delta f}{2 t_{m}}\left(\frac{2 R}{c}\right)^{2}-\frac{\Delta f}{t_{m}}\left(\frac{2 R}{c}\right) t \\
+\frac{A_{n}}{2 \pi f_{n}}\left[\cos 2 \pi f_{n}\left(t-\frac{2 R}{c}\right)-\cos 2 \pi f_{n} t\right]
\end{array}\right\}
$$

where $c_{0}$ is the signal amplitude, $c$ is the light velocity, $f_{0}$ is the center frequency, $\Delta f$ is the modulation bandwidth, $R$ is the range between an object and the radar, $t_{m}$ is the chirp length, $A_{n}$ is the amplitude noise component, and $f_{n}$ is the frequency noise component. The radar parameters for beat signal generation in Eq. (3) are $f_{0}$ of $76.5 \mathrm{GHz}, \Delta f$ of $200 \mathrm{MHz}, R$ of $50 \mathrm{~m}, A_{n}$ of 0.01 , and $f_{n}$ of $1 \mathrm{MHz}$. To get the range FFT result, a beat signal in the time domain in Eq. (3) is sampled and transformed into the frequency domain with a sampling frequency $f_{\mathrm{s}}$ of $10 \mathrm{MHz}$, a FFT size $N_{\text {FFT }}$ of 2048, and a Hann window. Note that applying a Hann window doubles the null-to-null bandwidth and $\Delta R$. Here, $\mathbf{s}$ and $\mathbf{y}$ in Eq. (2) can be generated from Eq. (3) with the range parameters and range FFT. The $\mathbf{s}$ is the 


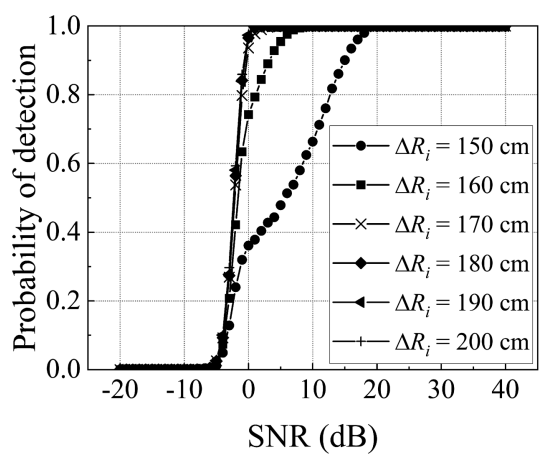

(a)

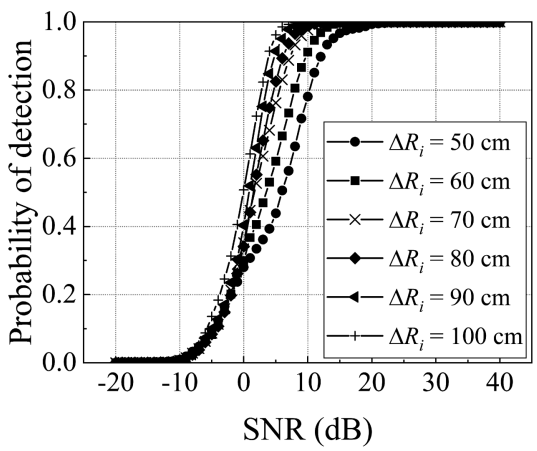

(b)

Fig. 3. Simulated probabilities of two-object detection (a) Conventional range detection (b) Proposed range detection

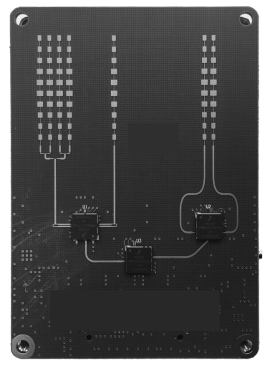

(a)
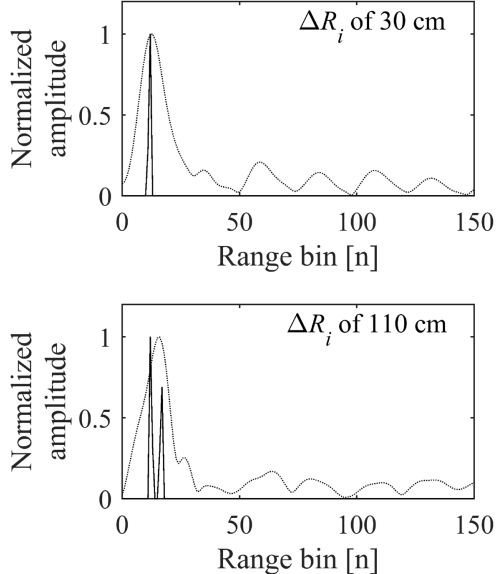

Fig. 4. Measurement environment and results (a) Radar module (b) and post-processing (lined) outputs

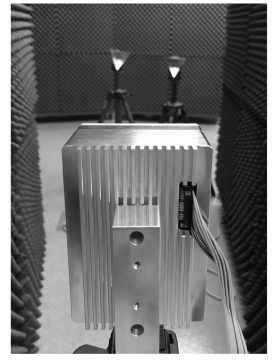

(b)
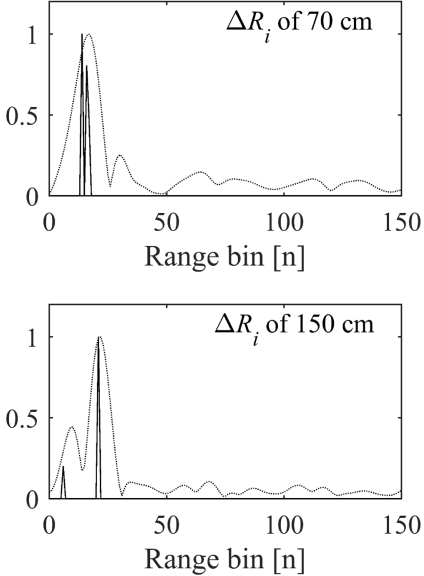

c)
Radar module and two corner reflectors (c) Range FFT (dotted)

normalized main-lobe magnitude components of a beat signal for one object without additive white Gaussian noise (AWGN), while $\mathbf{y}$ can be generated by summing the beat signals for each object with AWGN. For simulations without and with the application of the proposed post-processing method, the range interval $\Delta R_{i}$ between two objects was varied from 150 to $200 \mathrm{~cm}$ and from 50 to $100 \mathrm{~cm}$ with $10 \mathrm{~cm}$ intervals, respectively, and the SNR was varied from -20 to $40 \mathrm{~dB}$ with $1 \mathrm{~dB}$ intervals. Threshold values were set to satisfy the probability of false alarm $\left(P_{F A}\right)$ of $10^{-3}$ for each SNR [1]. 
Fig. 3 shows the simulated detection probabilities $\left(P_{D}\right)$ for distinguishing and detecting two objects for each $\Delta R_{i}$. Fig. 3(a) shows the $P_{D}$ for the conventional range FFT, while Fig. 3(b) shows the $P_{D}$ when the proposed post-processing method is applied after the range FFT to compensate for the deterioration of the range resolution. When the $P_{D}$ of 0.9 and $P_{F A}$ of $10^{-3}$ required in a typical radar system are satisfied [8], the $\Delta R$ is $160 \mathrm{~cm}$ due to the Hann window effect and nonlinearity noise as shown in Fig. 3(a); however, the improved $\Delta R$ becomes $70 \mathrm{~cm}$ as a result of post-processing based on the non-negative LSM as shown in Fig. 3(b). Note that the theoretical $\Delta R$ is $75 \mathrm{~cm}$ with the signal bandwidth of $200 \mathrm{MHz}$ according to Eq. (1).

Fig. 4 shows the measurement environment and results. Fig. 4(a) shows the $77 \mathrm{GHz}$ radar module, which was implemented using a NXP transceiver chipset (MR2001TX/RX/VC), NXP MCU (MPC 5775K), and planar antennas. Fig. 4(b) shows the measurement environment. One corner reflector was located at $1.3 \mathrm{~m}$ and the other corner reflector was located with $\Delta R_{i}$ to verify the $\Delta R$ in Fig. 3(b). The two corner reflectors were designed to meet a radar cross section (RCS) of $10 \mathrm{dBsm}$ at $77 \mathrm{GHz}$. Fig. 4(c) shows the range FFT (dotted) and post-processing (lined) outputs of the measured beat signals with the SNR of $21 \mathrm{~dB}$, when $\Delta R_{i}$ is 30,70 , 110 , and $150 \mathrm{~cm}$, respectively. When $\Delta R_{i}$ was less than $150 \mathrm{~cm}$, the beat frequency components corresponding to two objects overlapped in the range FFT result. However, when $\Delta R_{i}$ was $70 \mathrm{~cm}$ or more, by applying the post-processing to the range FFT result, the frequency components could be distinguished and appeared at each range bin with an average error of $31 \mathrm{~cm}$. Note that the range bin resolution is $13.8 \mathrm{~cm}$.

\section{Conclusion}

The proposed post-processing method is applied to range FFT results to improve a degraded the range resolution $\Delta R$ due to non-ideal factors in FMCW radar. The proposed method derives a high-resolution range profile in frequency range from a measured beat signal and predicted beat signals reflecting deterioration information, based on the non-negative LSM. To verify the proposed method, beat signals for two objects with the range interval $\Delta R_{i}$ of $70 \mathrm{~cm}$ were measured from the radar module with $f_{0}$ of $76.5 \mathrm{GHz}$ and $\Delta f$ of $200 \mathrm{MHz}$. The two objects with $\Delta R_{i}$ of $70 \mathrm{~cm}$, which are difficult to distinguish by conventional range detection, can be distinguished and detected by applying the proposed post-processing method.

\section{Acknowledgments}

This work was supported by Electronics and Telecommunications Research Institute (ETRI) grant funded by the Korean government. [18ZD1120, Development of ICT Convergence Technology for Daegu-GyeongBuk Regional Industry] 